\title{
Analysis of thermal effects in endoscopic nanocarriers-based Photodynamic Therapy applied to esophageal diseases
}

\author{
I. Salas-García ${ }^{\mathrm{a}}$, F. Fanjul-Vélez ${ }^{\mathrm{a}}$, N. Ortega-Quijano ${ }^{\mathrm{a}}$, O. Wilfert ${ }^{\mathrm{b}}$, L. Hudcova ${ }^{\mathrm{b}}$, J. Poliak $^{\mathrm{b}}$, P. \\ Barcik $^{\mathrm{b}}$, J. L. Arce-Diego ${ }^{*}$ \\ ${ }^{a}$ Applied Optical Techniques Group, TEISA Department, University of Cantabria \\ Av. de los Castros S/N, 39005 Santander (Spain); \\ ${ }^{\mathrm{b}}$ Institute of Radio Electronics, Brno University of Technology, Purkynova 118, 61200 Brno, Czech \\ Republic
}

\begin{abstract}
In this work we propose a predictive model that allows the study of thermal effects produced when the optical radiation interacts with an esophageal or stomach disease with gold nanoparticles embedded. The model takes into account light distribution in the tumor tissue by means of a Monte Carlo method. Mie theory is used to obtain the gold nanoparticles optical properties and the thermal model employed is based on the bio-heat equation. The complete model was applied to two types of tumoral tissue (squamous cell carcinoma located in the esophagus and adenocarcinoma in the stomach) in order to study the thermal effects induced by the inclusion of gold nanoparticles.
\end{abstract}

Keywords: esophageal tumor, photodynamic therapy, gold nanoparticles, photo-thermal interaction, endoscopic PDT

\section{INTRODUCTION}

The incidence of superficial squamous cell carcinoma in the esophagus and high grade dysplasia has steadily increased over the last decades. During this time Photodynamic Therapy (PDT) has become a promising treatment for these esophageal diseases mainly due to its noninvasive nature and the use of non ionizing radiation. However, its future viability as endoscopic mucosal treatment option requires the improvement of the present dosimetry [1]. Emerging strategies to improve the present treatment outcome include its combination with gold nanoparticles that act as carriers for conventional photosensitizers $[2,3]$. The inclusion of these nanoparticles in the pathological tissue could cause an increase in the optical radiation absorption that could lead to thermal damage in the target tissue.

In order to establish the proper dosimetry for Photodynamic Therapy, the use of predictive models constitutes a valuable tool $[4,5]$. Here we present a predictive model to estimate the thermal effects induced by gold nanoparticles combined with PDT for two types of malignant tissues. The light and temperature distribution in these tissues with gold nanoparticles embedded are modeled in section 2 . The results obtained are presented in the next section. Finally the conclusions obtained are listed in section 4.

\section{MODEL}

\subsection{Optical propagation}

The heterogeneity of the biological tissue does not allow an analytic exact approach of the radiation pattern with Maxwell equations. Radiation Transport Theory (RTT) can be used to obtain the distribution of light in a threedimensional tissue [6]. The model assumes that the scattering events are sufficiently numerous as to the light to be considered incoherent, in such a way that polarization or interference effects can be neglected. The basic parameter of light is the specific intensity $I(\vec{r}, \hat{s})$ defined as the light power per unit area per unit solid angle. The radiation is expected to be at the point $\vec{r}$, and to follow the direction $\hat{s}$. The scattering events are treated according to the scattering phase function $p\left(\hat{s} \cdot \hat{s}^{\prime}\right)$, which contains the probabilities of light to be scattered in the different directions. Optical radiation comes from direction $\hat{s}^{\prime}$ and is redirected to $\hat{s}$. The basic idea in order to write the differential radiation

* arcedj@unican.es; phone +34 9422015 45; www.teisa.unican.es/toa 
transport equation is that radiation from a particle attenuates due to absorption and scattering and also gains power because another particle can scatter light in the direction of the particle of interest. Therefore the differential radiation transport equation can be written with no sources inside the tissue and in a steady-state situation as in (1).

$$
\hat{s} \cdot \bar{\nabla} I(r, \hat{s})=-\left(\mu_{a}+\mu_{s}\right) I(r, \hat{s})+\frac{\mu_{s}}{4 \pi} \int_{4 \pi} p\left(\hat{s}^{\prime} \cdot \hat{s}^{\prime}\right) I\left(r, \hat{s}^{\prime}\right) d \Omega^{\prime}
$$

The Monte Carlo method has demonstrated its applicability and accuracy for the numerical analysis of the radiation transport equation compared with the exact solutions. Among the different implementations of the Monte Carlo method applied to the RTT model, the most used is probably the one by Wang and Jacques, [7, 8]. This implementation of the Monte Carlo model is also multi-layered, so it is possible to define several layers of different materials with their borders perpendicular to the laser beam, which is very useful due to tissues can be usually divided in different strata. Both tissue samples used in this work are divided in two layers. The most superficial corresponds to the tumor tissue (squamous cell carcinoma or adenocarcinoma) and the other one to the esophagus and the stomach respectively. The optical characteristics and dimensions of each of the tissue layers are required to get a proper definition of the model. The optical parameters needed are the index of refraction $n$, the absorption coefficient $\mu_{a}$, the scattering coefficient $\mu_{s}$ and the anisotropy of scattering $g$. This last one is also known as the average cosine of scatter and is related with the scattering phase function. The average cosine of scatter gives an idea about the probability of being scattered in a particular direction.

In order to get the optical absorption and scattering properties of gold nanospheres of $80 \mathrm{~nm}$ radius, the efficiency factors of absorption $Q_{a b s}$, scattering $Q_{s c a}$ and extinction $Q_{\text {ext }}$, were calculated by means of Mie's theory for an homogeneous spherical nanoparticle with radius $r$ and the complex refractive index of gold $m$ [9]. The efficiency factors have a strong dependence with the optical radiation wavelength as well as with the nanoparticle size.

$$
\begin{gathered}
Q_{e x t}=\frac{2}{x^{2}} \sum_{n=1}^{\infty}(2 n+1) \operatorname{Re}\left(a_{n}+b_{n}\right) \\
Q_{s c a}=\frac{2}{x^{2}} \sum_{n=1}^{\infty}(2 n+1)\left(\left|a_{n}\right|^{2}+\left|b_{n}\right|^{2}\right) \\
Q_{a b s}=Q_{e x t}+Q_{a b s}
\end{gathered}
$$

The amplitude coefficients $a_{n}$ and $b_{n}$ for the scattered field have been calculated by the expressions (5) and (6), which involve spherical Bessel functions [10], the refractive index of the sphere relative to the ambient medium $m, x=k r$ is the size parameter, $k=2 \pi / \lambda$ is the wave number, $\lambda$ is the wavelength in the ambient medium and $\mu_{1}$ is the ratio of the magnetic permeability of the sphere to the magnetic permeability of the ambient medium.

$$
\begin{gathered}
a_{n}=\frac{m^{2} j_{n}(m x)\left[x j_{n}(x)\right]^{\prime}-\mu_{1} j_{n}(x)\left[m x j_{n}(m x)\right]^{\prime}}{m^{2} j_{n}(m x)\left[x h_{n}^{(1)}(x)\right]^{\prime}-\mu_{1} h_{n}^{(1)}(x)\left[m x j_{n}(m x)\right]^{\prime}} \\
b_{n}=\frac{\mu_{1} j_{n}(m x)\left[x j_{n}(x)\right]^{\prime}-j_{n}(x)\left[m x j_{n}(m x)\right]^{\prime}}{\mu_{1} j_{n}(m x)\left[x h_{n}^{(1)}(x)\right]^{\prime}-h_{n}^{(1)}(x)\left[m x j_{n}(m x)\right]^{\prime}}
\end{gathered}
$$

Nanoparticle absorption and scattering coefficients are quantified from the efficiency parameters previously shown, the cross section of the spherical nanoparticle and the number of nanoparticles per unit volume $N_{T}$ by the expressions (7) and (8) respectively.

$$
\mu_{a b s}=\pi r^{2} Q_{a b s} N_{T}
$$




$$
\mu_{s c a}=\pi r^{2} Q_{s c a} N_{T}
$$

\subsection{Thermal modeling}

The study of the thermal effects produced by PDT combined with gold nanoparticles is carried out from the optical radiation absorption in each point of the tissue sample. The main heat mechanisms that will be considered are [11]: conduction, defined as the diffusion of heat in a solid from hot regions to colder ones; the convection resulting from the interaction between a solid surface and a fluid in contact with it; and the radiation or heat transport due to the infrared emission of a body at a nonzero temperature. In order to obtain a differential equation that describes the spatial and temporal behavior of the tissue temperature (i.e., the bio-heat equation) we first take the balance equation for the thermal energy as expressed in (9). Then, each element of (9) admits an integral expression giving the final expression (10).

$$
\begin{gathered}
Q_{\text {gain }}=Q_{\text {storage }}+Q_{\text {loss }} \\
\int_{V} q(\vec{r}, t) d V=\int_{V} \rho c \frac{d T(\vec{r}, t)}{d t} d V-\int_{S} k \nabla T(\vec{r}, t) \hat{n} d S-\int_{V} \rho_{b} c_{b} w_{b}\left[T_{\text {art }}(\vec{r}, t)-T_{\text {ven }}(\vec{r}, t)\right] d V
\end{gathered}
$$

Where $q(\vec{r}, t)$ is the volumetric heat generated at position $\vec{r}$ at time $t$. The absolute temperature is given by $T$, in a tissue of density $\rho\left[\mathrm{kg} / \mathrm{m}^{3}\right]$ and specific heat $c[\mathrm{~J} / \mathrm{Kg} \cdot \mathrm{K}]$. It is assumed that the internally generated heat is negligible. Parameter $k[\mathrm{~W} / \mathrm{m} \cdot \mathrm{K}]$ is the thermal conductivity, that governs the losing effect of conduction. The convection effect caused by blood perfusion in living tissues is modeled by the volumetric perfusion $w_{b}\left[\mathrm{~m}_{\mathrm{b}}{ }^{3} / \mathrm{s} \cdot \mathrm{kg}_{\text {tissue }}\right]$, as well as by the venous and arterial temperatures. The resolution of the bio-heat equation is performed by the finite difference method, so that the temperature distribution in space and time can be obtained. It should be remarked that the boundary conditions must be carefully defined. The model comprises a wide range of effects and entails a considerable complexity, giving a realistic approach for the study of the thermal effects in biological tissues.

\section{RESULTS AND DISCUSSION}

The scattering and absorption coefficients dependence with the wavelength of the optical radiation applied, is shown in Fig. 1 for gold nanoparticles of $80 \mathrm{~nm}$ radius and an homogeneous density of $2 \cdot 10^{9} \mathrm{~cm}^{-3}$ in the tissue sample. As it can be observed both the election of the proper scattering and absorption coefficients depends on the wavelength of the application we are dealing with, $630 \mathrm{~nm}$ in Photodynamic Therapy with the photosensitizer Methyl Aminolevulinate Protoporphyrin IX (MAL-PpIX).

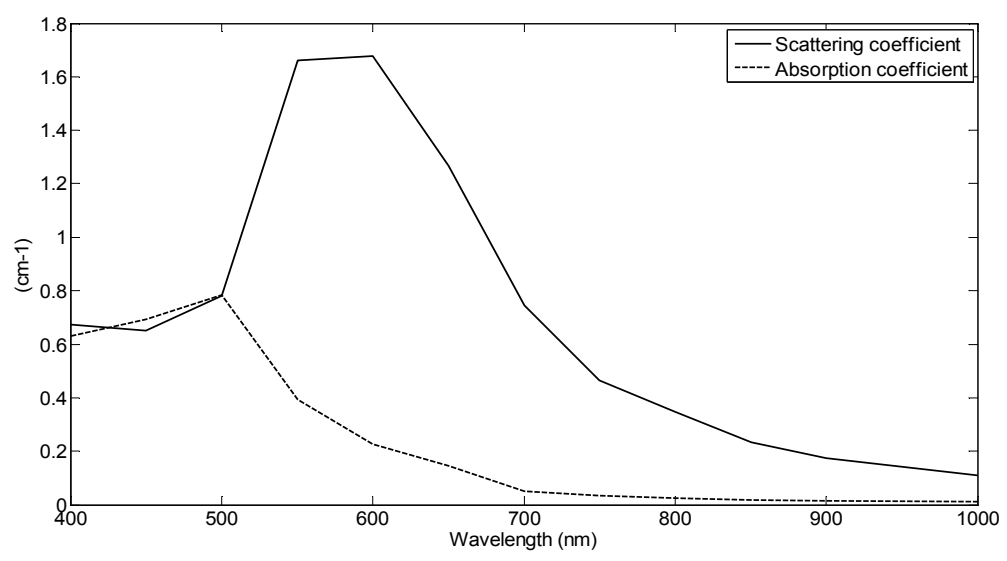

Figure 1. Gold nanoparticles optical properties dependence with the wavelength of the optical radiation applied.

The optical absorption values were obtained for two different types of malignant pathologies, a squamous cell carcinoma located in the esophagus wall and an adenocarcinoma in the stomach, whose optical properties are summed up in Table 1 [12]. 
Table 1. Optical properties at $630 \mathrm{~nm}$ used for each layer of the tissue samples employed and their depth.

\begin{tabular}{|c|c|c|c|c|c|}
\hline Layer & $\begin{array}{c}\text { Absorption } \\
\text { coefficient } \\
\left(\mathrm{mm}^{-1}\right)\end{array}$ & $\begin{array}{c}\text { Scattering } \\
\text { coefficient } \\
\left(\mathrm{mm}^{-1}\right)\end{array}$ & Anisotropy & $\begin{array}{c}\text { Index of } \\
\text { refraction }\end{array}$ & $\begin{array}{c}\text { Depth } \\
(\mathrm{mm})\end{array}$ \\
\hline SCC & 0.13 & 9.35 & 0.92 & 1.37 & 2 \\
\hline Esophagus & 0.21 & 12.56 & 0.94 & 1.37 & 1 \\
\hline Adenocarcinoma & 0.16 & 12.99 & 0.94 & 1.37 & 1 \\
\hline Stomach & 0.26 & 13.96 & 0.94 & 1.37 & 1 \\
\hline
\end{tabular}

A cylindrical laser beam with a radius of $0.1 \mathrm{~cm}$ perpendicular to the tissue sample was used to deliver an optical power of $0.04 \mathrm{~W}$. The tissue sample was composed of two layers. The upper one was the malignant tissue which lied on healthy tissue, esophagus and stomach, respectively. Figure 2 shows the results obtained in both cases with and without nanoparticles embedded in the tumoral tissue. In both types of tissues, the inclusion of gold nanoparticles produced an increase in the optical absorption.
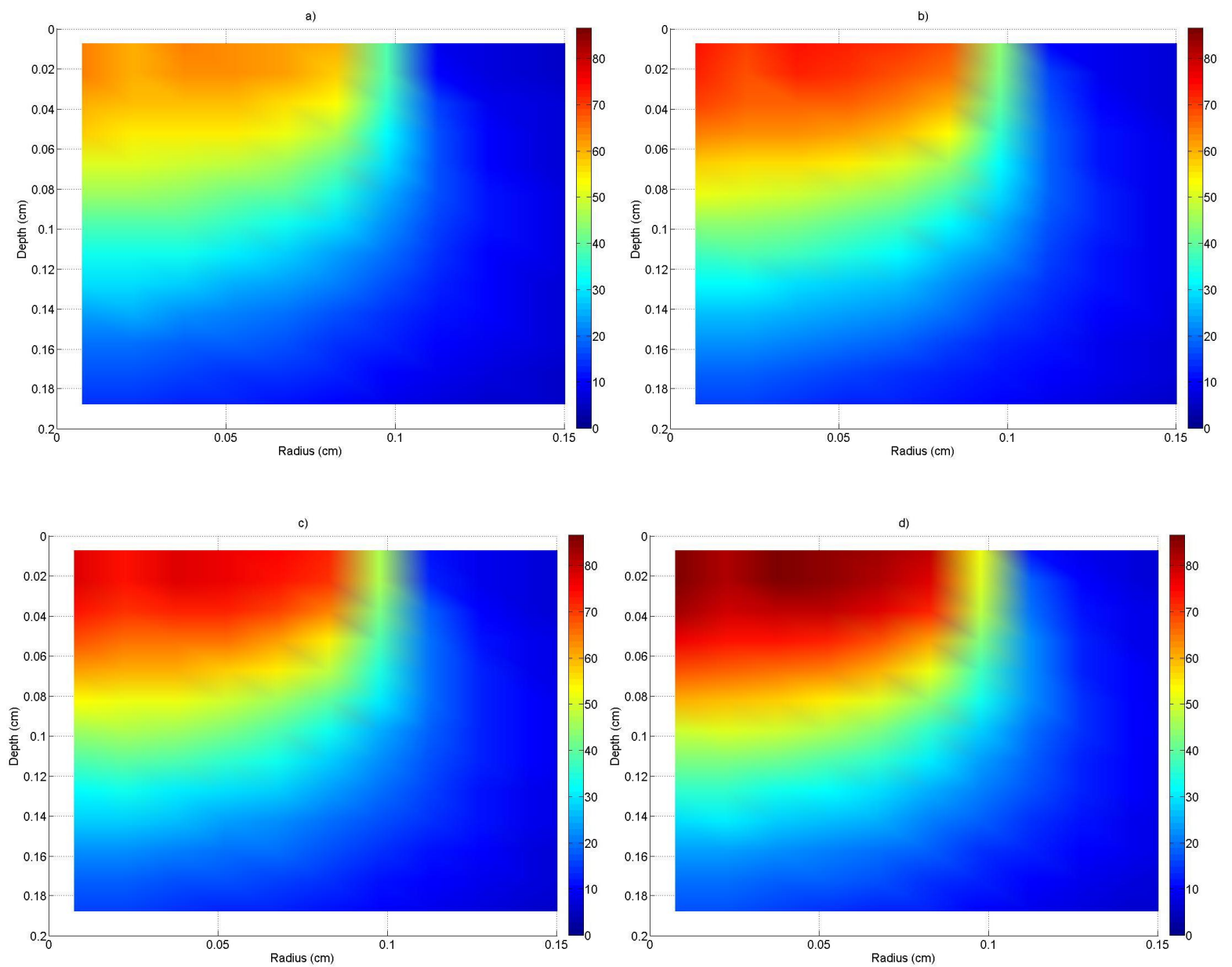

Figure 2. Optical absorption $\left(\mathrm{J} / \mathrm{cm}^{3}\right)$ for a squamous cell carcinoma in the esophagus without a) and with b) nanoparticles and for an adenocarcinoma in the stomach without c) and with d) nanoparticles. 
These results were used to calculate the temperature distribution in the volume occupied by the tumor tissue assuming an initial body temperature of $37{ }^{\circ} \mathrm{C}$. The final temperature reached in all the cases is depicted in Fig. 3 vs. the radius and the depth in the tissue sample. The maximum temperatures reached were $37.1090{ }^{\circ} \mathrm{C}, 37.1251{ }^{\circ} \mathrm{C}, 37.1344{ }^{\circ} \mathrm{C}$ and $37.1502{ }^{\circ} \mathrm{C}$. As it can be observed, there was always a negligible increase in the local temperature when the nanoparticles were administered. Therefore the increase in the optical absorption occurred as a result of the administration of the gold nanoparticles employed has no significant effect on tissue heating. This last issue makes these gold nanoparticles suitable to act as photosensitizer carriers for the conventional power densities used in photodynamic therapy, although they will not improve the photodynamic therapy results by inducing thermal effects.
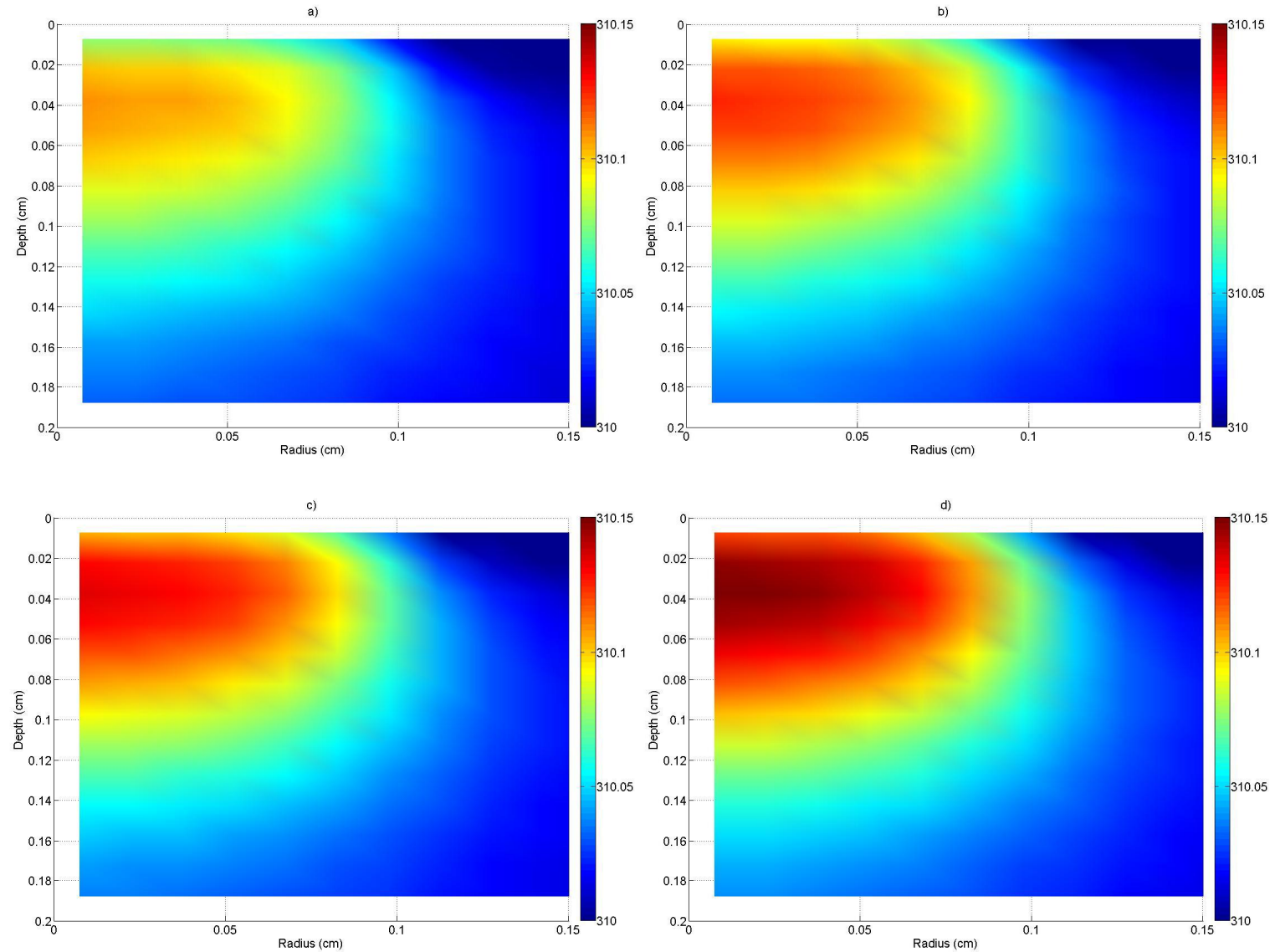

Figure 3. Temperature distribution (K) for a squamous cell carcinoma in the esophagus without a) and with b) nanoparticles and for an adenocarcinoma in the stomach without c) and with d) nanoparticles.

\section{CONCLUSIONS}

A predictive model was applied to obtain the temperature distribution in two types of malignant tissues located in the esophagus and stomach wall, respectively, which are subjected to photodynamic therapy combined with a uniform distribution of gold nanoparticles embedded. The optical properties of gold spherical nanoparticles were obtained by means of Mie theory. Light propagation in the tissue was described using a numerical Monte Carlo model. The optical absorption values obtained were used to calculate the temperature distribution in the volume occupied by the tumor tissue by the bio-heat equation. The administration of gold nanoparticles to a squamous cell carcinoma and an adenocarcinoma showed a negligible increase in the local temperature using an optical power commonly applied in photodynamic therapy with the photosensitizer MAL-PpIX. Although they will not contribute to the improvement of the photodynamic effects by triggering a thermal interaction that leads to thermal effects, $80 \mathrm{~nm}$ gold nanoparticles could be suitable to act as MAL carriers for photodynamic therapy. 


\section{ACKNOWLEDGEMENTS}

This work has been partially supported by the project MAT2012-38664-C02-01 of the Spanish Ministery of Economy and Competitiveness, by the Czech Grant Agency under grant P102/11/1376, by the Czech Ministry of Industry and Trade under grant FR-TI2/705, by the Czech Ministry of Education under grant LD12067, and by the EU COST Action IC1101.

\section{REFERENCES}

[1] Bays, R., Wagnières, G., Robert, D., Braichotte, D., Savary, J. F., Monnier, P. and van den Bergh, H., "Light Dosimetry for Photodynamic Therapy in the Esophagus" Lasers in Surgery and Medicine 20, 290-303 (1997).

[2] Hamblin M. R. and Mróz P., [Advances in Photodynamic Therapy. Basic, translational and clinical], Engineering in medicine \& Biology, Hamblin Mróz editors, 1-239 (2008).

[3] Allison, R.R., Mota, H.C., Bagnato, V.S. and Sibata, C.H., "Bio-nanotechnology and photodynamic therapy: State of the art review" Photodiagnosis and Photodynamic Therapy 5, 19-28 (2008).

[4] Hu, X. H, Feng, Y., Lu, J. Q., Allison, R. R., Cuenca, R. E., Downie, G. H. and Sibata, C. H., "Modeling of a type II Photofrin-mediated PDT process in a heterogeneous tissue phantom" Photochemistry and Photobiology 81, 1460-1468 (2005).

[5] Salas-García, I., Fanjul-Vélez, F., Arce-Diego, J. L., "Photosensitizer absorption coefficient modeling and necrosis prediction during Photodynamic Therapy" Journal of Photochemistry and Photobiology B: Biology 114, 79-86 (2012).

[6] Vo-Dinh, T., [Biomedical Photonics Handbook], T. Vo-Dinh, ed., CRC Press, Boca Raton, (2003).

[7] Wang, L., Jacques, S. L. and Zheng, L., "MCML - Monte Carlo modeling of light transport in multi-layered tissues" Computer methods and programs in biomedicine 47, 131-146 (1995).

[8] Wang, L., Jacques, S. L. and Zheng, L., "CONV - Convolution for responses to a finite diameter photon beam incident on multi-layered tissues" Computer methods and programs in biomedicine 54, 141-150 (1997).

[9] Bohren, C.F. and Huffman, D.R., [Absorption and Scattering of Light by Small Particles], John Wiley, New York, NY (1983).

[10] Mätzler, C., "MATLAB Functions for Mie Scattering and Absorption”, Universitas Bernensis, (2002).

[11]Fanjul-Vélez, F., Romanov, O. G., Arce Diego, J. L., "Efficient 3D numerical approach for temperature prediction in laser irradiated biological tissues" Computers in Biology and Medicine 39, 810-817 (2009).

[12] Holmer, C., Lehmann, K. S., Wanken, J., Reissfelder, C., Roggan, A., Mueller, G., Buhr, H. J., Ritz, J. P., "Optical properties of adenocarcinoma and squamous cell carcinoma of the gastroesophageal junction" Journal of Biomedical Optics 12(1), 014025-1-8 (2007). 